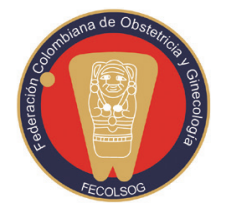

Revista Colombiana de Obstetricia y Ginecología Vol. 66 No. 1 • Enero-Marzo 2015 • (32-36)

\title{
PREVALENCIA DEL RESULTADO DE CITOLOGÍA DE CÉLULAS ESCAMOSAS ATÍPICAS QUE NO EXCLUYE LESIÓN INTRAEPITELIAL DE ALTO GRADO (ASC-H), EN DOS INSTITUCIONES DE BOGOTÁ (COLOMBIA), 2006-2013
}

\section{Prevalence of the result of atypical squamous cell citology which does not rulle out high grade intraepithelial lesion (ASC-H) in two institutions in Bogotá (Colombia), 2006-2013}

María Camila Hernández-Tiria, $M D^{I}$; Marcos Fidel Castillo-Zamora, MD $^{2}$

Recibido: mayo 26/14 - Aceptado: febrero 13/15

\section{RESUMEN}

Objetivo: determinar la prevalencia de citologías cérvico-vaginales con hallazgo citológico de tipo células escamosas atípicas que no descartan lesión escamosa intraepitelial de alto grado (ASC-H), en dos centros de patología cervical, y determinar en ellas la prevalencia de lesión escamosa intraepitelial de alto grado (LEI-AG) por biopsia.

Materiales y métodos: estudio de corte transversal en el cual se incluyen todas las pacientes que se practicaron citologías en dos centros de colposcopia en Bogotá: un hospital público y un centro de colposcopia privado, en el periodo comprendido entre 2006-2013. Se identificaron las citologías ASC-H, se realizó muestreo consecutivo, se evaluaron las características sociodemográficas de las pacientes atendidas, el resultado colposcópico y el resultado

1 Médica general, Universidad de La Sabana. Residente de Ginecología y Obstetricia, Universidad de La Sabana, Bogotá (Colombia).

2 Especialista en Ginecología y Obstetricia, Pontificia Universidad Javeriana. Profesor Asistente, Ginecología y Obstetricia, Universidad de La Sabana, Hospital Universitario de La Samaritana, Bogotá (Colombia). marcoscz@unisabana.edu.co de biopsia. En el análisis se presenta la prevalencia del resultado ASC-H, y sobre el número de pacientes con resultado de ASC-H se estimó la prevalencia de LEI-AG por histopatología.

Resultados: se identificaron 357 citologías ASC-H de 23.960 citologías anormales, calculando una prevalencia del 1,49\% (IC $95 \%$ : 1,34-1,64). Se dispuso de estudio histopatológico en 230 pacientes (65\%); en las pacientes con citología ASC-H la prevalencia de LEI-AG fue de 26,5\%.

Conclusiones: los resultados de este estudio mostraron una prevalencia de ASC-H del 1,49\%. Dado que un importante número de pacientes tienen lesión intraepitelial de alto grado, se recomienda la realización de biopsia dirigida por colposcopia en pacientes con estos resultados.

Palabras clave: displasia del cuello del útero, neoplasia intraepitelial del cuello uterino, citología, colposcopia.

\section{ABSTRACT}

Objective: To determine the prevalence of the finding of atypical squamous cells cannot 
exclude high-grade squamous intraepithelial lesions (ASC-H) in two pathology centres, and to determine the prevalence among those cytology findings of high grade squamous intraepithelial lesion (HSIL) on biopsy.

Materials and methods: Cross-sectional study including all patients undergoing cytology in two colposcopy centres in Bogotá, one in a public hospital and the other in a private centre, during the period between 2006 and 2013. ASC-H cytology results were identified in a sample of consecutive cases, and the assessment included the social and demographic characteristics of the patients and the colposcopy and biopsy results. The analysis focuses on the prevalence of the ASC-H result and of the HSIL result on histopathology.

Results: Overall, 357 ASC-H cytology results were identified out of 23,960 abnormal cytology findings, leading to an estimated prevalence of 1.49\% (95\% CI, 1.34-1.64). Histopathology was required in 230 cases (65\%) and the prevalence among them of HSIL was $26.5 \%$.

Conclusions: The results of this study showed a prevalence of $1.49 \%$ for ASC-H. Given that an important number of patients have a high-grade intraepithelial lesion, colposcopy-guided biopsy is recommended in patients with this result.

Key words: Cervical dysplasia, cervical intraepithelial neoplasia, cytology, colposcopy.

\section{INTRODUCCIÓN}

El cáncer de cuello uterino es el segundo en frecuencia y el de mayor mortalidad en el mundo, siendo solo superado por el cáncer de seno (1). Gracias a la existencia de un programa de tamización basado en la citología, sus tasas de incidencia y de mortalidad han disminuido durante los últimos años; sin embargo, la tasa de muerte por cáncer de cérvix sigue siendo alta, ha pasado de ser de 14 muertes por cada 100.000 mujeres en los años ochenta a 7,08 muertes por cada 100.000 en la actualidad (2).

La citología cervical reduce la incidencia de cáncer de cérvix ya que permite el diagnóstico de las lesiones precursoras del cáncer de cuello uterino. Se basa en que estas lesiones precancerosas producen células con diferentes grados de alteración en la maduración y diferenciación, que son detectables en el extendido celular (3). Las pacientes que presentan un resultado anormal son sometidas a una serie de exámenes complementarios tales como la colposcopia y la biopsia de cérvix para tener un diagnóstico histopatológico preciso (4). Con mayor frecuencia, el cáncer de cérvix se presenta en países en vías de desarrollo y su detección precoz es una alternativa costo-efectiva para reducir la morbimortalidad (5).

Según la Guía de Manejo de la American Society of Colposcopy and Cervical Pathology (ASCCP) para Mujeres con Anormalidades Citológicas de Cérvix, del 2012 (4), las lesiones histopatológicas de neoplasia intraepitelial cervical tipo 2 (NIC 2) y neoplasia intraepitelial cervical tipo 3 (NIC 3) podrían ser precedidas por un hallazgo citológico de tipo células escamosas atípicas que no descartan lesión escamosa intraepitelial de alto grado (ASC-H). Su hallazgo se ha vinculado histopatológicamente con la presencia de una lesión escamosa intraepitelial de alto grado (LEI-AG) (6). Es por esto que ante un reporte de ASC-H se recomienda referir la paciente a colposcopia $(7,8)$.

Pese a su relevancia clínica, poco se conoce con respecto a la prevalencia de ASC-H y de LEI-AG entre las pacientes con reporte citológico de ASC-H en nuestro medio.

El objetivo del presente estudio es describir la prevalencia de citología ASC-H y de LEI-AG entre las pacientes con reporte citológico de células escamosas atípicas, donde no se excluye lesión intraepitelial de alto grado en las mujeres que consultaron a dos instituciones de atención de patología cervical ubicadas en Bogotá (Colombia).

\section{MATERIALES Y MÉTODOS}

Se realizó un estudio de corte transversal en el cual se incluyen todas las pacientes con citologías tomadas en el periodo de tiempo comprendido en- 
tre 2006 al 2013, en dos centros de colposcopia en Bogotá, uno de los cuales es un hospital público de segundo nivel y el segundo es una institución prestadora de salud privada, que atienden tanto régimen contributivo como subsidiado del sistema seguridad social en Colombia. Se excluyeron las pacientes con citologías no aptas para lectura.

Previo diseño de un instrumento para la recolección de la información, los investigadores revisaron la base de datos de las dos instituciones participantes donde se identificaron los casos que tuvieran resultado citológico de ASC-H. Se registraron la edad, el resultado citológico, el régimen de seguridad social, el resultado colposcópico y el resultado de biopsia en las pacientes con citología ASC-H.

Para el análisis de la información se utilizó el programa estadístico SPSS Versión 17, y se realizó la estadística descriptiva de las variables. Se obtuvieron frecuencias absolutas y relativas para las variables categóricas y la media, y desviación estándar y rango para las variables continuas. Sobre el total de citologías, se estimó la prevalencia del ASC-H, y sobre el número de pacientes con resultado de ASC-H se estimó la prevalencia de LEI-AG y lesión escamosa intraepitelial de bajo grado (LEI- BG).

Aspectos éticos. Este estudio respetó la confidencialidad de las pacientes y fue aprobado por el comité de ética de las instituciones.

\section{RESULTADOS}

Durante periodo de estudio se realizaron 23.960 citologías en pacientes, y de ellas 357 fueron reportadas como ASC-H, para una prevalencia estimada de 1,49\% citologías con este hallazgo.

La edad media de las pacientes con ASC-H fue de 44,7 años, con una desviación estándar de 14,5. El 65,3\% pertenecían al régimen contributivo y el 24,6\% al régimen subsidiado. El $5 \%$ accedió al servicio de salud con recursos propios o no contaban con cobertura al momento del estudio. Al 10,9\% se le realizó el diagnóstico de LEI-AG durante la colposcopia, en tanto que se hizo el diagnóstico colposcópico de LEI-BG al 21,8 \%.
De las 357 pacientes con citología ASC-H, a 230 pacientes (65\%) se les realizó biopsia de cérvix con resultado de patología. Los resultados anatomopatológicos del total de muestras tomadas fueron: LEI-AG, 26,5 \%; LEI-BG, lesión de bajo grado, $14,7 \%$; adenocarcinoma, 0,86\%; carcinoma invasor, 3,47\%; VPH, 1,7\%. En el 52,6\% de todas las biopsias tomadas los resultados fueron reportados como negativos para lesión intraepitelial.

\section{DISCUSIÓN}

La prevalencia de ASC-H en nuestro estudio fue de 1,49\%, y la prevalencia de lesión de alto grado fue del $26,5 \%$ en el grupo de mujeres con citología ASC-H en las que se dispuso de material histopatológico.

Nuestros resultados discrepan de la prevalencia de ASC-H publicada por otros autores. El estudio realizado por Barreth et al. (9) reportó una frecuencia de 727 resultados ASC-H de un total de 241.841 citologías, para una prevalencia de este resultado del 0,3\%. Elsheick et al. informan una frecuencia del ASC-H del 0,15\% en un total de 129.911 citologías (10); por otra parte, se ha descrito que en general su frecuencia está cercana al $1 \%$ (11). La frecuencia del citología ASC-H informada por nosotros podría haber estado sobreestimada dado que son clínicas de patología cervical donde se concentran pacientes con resultados anormales de citología o sesgo de referencia. Sin embargo, se debe tomar en cuenta que por el tipo de citología utilizada la frecuencia podría haber sido ligeramente menor a la informada por estudios en que la citología fue con base líquida, técnica que presenta una mayor sensibilidad en contraste con la muestra de Papanicolaou convencional utilizada de forma estándar en nuestro medio. Se ha informado una sensibilidad de $71 \%$ con una especificidad del $98 \%$ para Papanicolaou frente un 80,1 y 97,2\% respectivamente con base líquida (12).

Respecto al hallazgo de neoplasia intraepitelial de alto grado en pacientes con citologías ASC-H, nuestros resultados son similares a los reportados por Nogara et al., quienes informan una prevalen- 
cia de LIE-AG de 23,7\% en mujeres con citología ASC-H sometidas a procedimiento de escisión por electrocirugía (LEEP) (13); Srodon et al. informan una frecuencia de LEI-AG del $40 \%$ (14), similar a la reportada por Elsheikh et al. (10) y menor a la informada por Sherman et al., del $80 \%$ (15) y por Kietpeerakool et al., del $61 \%$ (7). El no haber contado con el resultado de patología en el $35 \%$ de las pacientes con citología ASC-H, podría haber llevado a una importante subestimación de la frecuencia de lesiones de alto grado (NIC II y NICIII) en esta serie de pacientes.

\section{CONCLUSIÓN}

Los resultados de este estudio mostraron una prevalencia de ASC-H del 1,49\%. Dado que un importante número de pacientes tienen lesión intraepitelial de alto grado, se recomienda la realización de biopsia dirigida por colposcopia en pacientes con este resultado.

\section{REFERENCIAS}

1. Ochoa Jaramillo FL, Montoya Vélez LP. Mortalidad por cáncer en Colombia. Revista CES Medicina. 2004;18:19-36.

2. Ministerio de Salud y Protección Social. Bajan tasas de incidencia y mortalidad de cáncer de cuello uterino en Colombia, 16/09/2013. Boletín de Prensa 310; 2013. [Visitado 2014 Dic 31]. Disponible en: http:// www.minsalud.gov.co/Paginas/Bajan-tasas-incidenciamortalidad-cancer-cuello.aspx

3. Wright TC Jr. Pathology of HPV infection at the cytologic and histologic levels: Basis for a 2-tiered morphologic classification system. International Journal of Gynecology and Obstetrics. 2006;94:S22-S31.

4. Stewart L, Einstein M, Huh W. 2012 updated consensus guidelines for the management of abnormal cervical cancer screening tests and cancer precursors for the 2012 ASCCP consensus guidelines conference. Journal of Lower Genital Tract Disease. 2103;17:S1-S27.

5. American Cancer Society. Cancer Facts \& Figures 2012. Atlanta; 2012.

6. Barcelos AC, Michelin MA, Adad SJ, Murta EF.
Atypical squamous cells of undetermined significance: Bethesda classification and association with Human Papillomavirus. Infect Dis Obstet Gynecol. 2011; 2011:904674.1-9 doi: 10.1155/2011/904674.

7. Kietpeerakool C, Srisomboon J, Tantipalakorn C, Suprasert P, Khunamornpong S, Nimmanhaeminda K et al. Underlying pathology of women with "atypical squamous cells, cannot exclude high-grade squamous intraepithelial lesion" smears, in a region with a high incidence of cervical cancer. J Obstet Gynaecol Res. 2008;34:204-9.

8. Wright TC Jr, Cox JT, Massad LS, Twiggs LB, Wilkinson EJ. ASCCP-Sponsored Consensus Conference. 2001 Consensus Guidelines for the management of women with cervical cytological abnormalities JAMA. 2002; 287:2120-9.

9. Barreth D, Schepansky A, Capstick V, Johonson G, Steed H, Faught W. Atypical squamous cells, cannot exclude high-grade squamous intraepithelial lesion (ASC-H): a result not to be ignored. J Obstet Gynaecol Can. 2006;28:1095-8.

10. Elsheikh TM, Kirkpatrick JL, Wu HH. The significance of "low-grade squamous intraepithelial lesion, cannot exclude high-grade squamous intraepithelial lesion” as a distinct squamous abnormality category in Papanicolaou tests. Cancer. 2006;108:277-81.

11. Davey DD, Greenspan DL, Kurtycz DF, Husain M, Austin RM. Atypical squamous cells, cannot exclude high-grade squamous intraepithelial lesion: review of ancillary testing modalities and implications for follow-up. J Low Genit Tract Dis. 2010;14:206-14.

12. Cox JT. Liquid-base cytology: evaluation of effectiveness, cost-effectiveness, and application to present practice. J Natl Compr Canc Netw. 2004;2:597-611.

13. Nogara PR, Manfroni LA, Consolaro ME. Cervical cytology of atypical squamous cells cannot exclude high-grade squamous intraepithelial lesion (ASC-H): histological results and recurrence after a loop electrosurgical excision procedure. Arch Gynecol Obstet. 2011;284:965-71.

14. Srodon M, Parry Dilworth H, Ronnett BM. Atypical squamous cells, cannot exclude high-grade squamous intraepithelial lesion: diagnostic performance, human 
papillomavirus testing, and follow-up results. Cancer. 2006;108:32-8.

15. Sherman ME, Castle PE, Solomon D. Cervical cytology of atypical squamous cells-cannot exclude high-grade squamous intraepithelial lesion (ASC-H): characteristics and histologic outcomes. Cancer. 2006;108:298-305. 